COMMUNICATIONS IN

ANALYSIS AND GEOMETRY

Volume 12, Number 5, 1009-1037, 2004

\title{
Moment Map, Futaki Invariant and Stability of Projective Manifolds
}

\begin{abstract}
XIAOWEI WANG
In this paper, by applying the theory of moment map we provide an unified picture of stability of projective manifolds. In particular, a new integral formula of evaluating Chow weight is given and a simple way of constructing unstable manifolds is presented.
\end{abstract}

\section{Introduction.}

Let $(X, L)$ be a projective manifold polarized by an ample line bundle $L$, the problem of finding necessary and sufficient condition for the existence of a Kähler metric with constant scalar curvature in the Kähler class $\left[c_{1}(L)\right]$ has attracted people for decades. In mid 80's, Futaki introduced the first integral obstructions ( Futaki invariants) to the existence of constant scalar curvature metric based on the similar result on the integral obstructions to the prescribing scalar curvature problem in Riemannian geometry found earlier by Kazdan and Warner. Since then there have been a lot of study on this type of obstructions, a good account for this is Futaki's book [F]. In particular, Yau have conjectured that the GIT stability of the underlying manifold must provided further obstruction to the existence of the constant scalar curvature metric.

In this note, we use the theory of moment map to give a new and natural interpretation of the obstructions to stability of polarized manifolds. As applications, first, in section 3 , we present a new and differential geometric way of evaluating the Chow weight (Theorem 26); as a corollary, a new and algebro-geometric proof of Zhang's theorem ([Z]) on the equivalence between the balanced condition and Chow poly-stability of a projective embedding (Corollary 27) is given. Second, in section 4 we introduce a family of finite dimensional obstructions which gives an algebraic geometric interpretation of Futaki character (Theorem 38), and as a byproduct, it explains why Futaki invariants also arise as the obstruction to the Chow semi-stability of $X$ with respect to $L$, in particular, these results provide supporting evidence to Yau's conjecture. A similar result was found earlier by Mabuchi and Nakagawa 
[MN]. Third, in section 5 by analyzing the symplectic geometric aspect of Hilbert and Chow scheme, we are able to give a simple way of constructing examples of unstable manifolds, in particular they admit no constant scalar curvature metric by the recent results of Donaldson. These manifolds include the Mukai-Tian manifolds and examples due to Burns and De Bartolomeis.

Acknowledgement: I am very grateful to Patrick Bronsnan, David Gieseker, Amalendu Krishna, Conan Leung, Kefeng Liu, Julius Ross and Richard Thomas for many very helpful discussions and comments, and to professor S.-T. Yau for his constant encouragement. The author also wants to thank the referee for his many helpful comments.

\section{Symplectic quotient.}

In this section we collect elementary facts of the symplectic quotient and GIT quotient that will be used later. Let us first fix the notation, throughout this section $(\mathcal{Z}, L, \omega)$ will be a Kähler manifold(possibly infinite dimensional) polarized by an ample line bundle $\pi: L \rightarrow \mathcal{Z}$ with a Hermitian metric $h$ such that $\omega=\frac{i}{2 \pi} \operatorname{Ric}(h)$.

\subsection{Symplectic quotient versus GIT.}

We first recall the interplay between symplectic quotient and GIT quotient in finite dimensional case. Throughout this subsection we assume that $(\mathcal{Z}, L, \omega)$ is a finite dimensional projective manifold polarized by ample line bundle $L$. Let $G$ be a compact Lie group acting on $\mathcal{Z}$ by holomorphic isometry, then the $G$ action can be naturally extended to a $G^{\mathbb{C}}$ action on $\mathcal{Z}$, where $G^{\mathbb{C}}$ is the complexification of $G$. Let $\langle\cdot, \cdot\rangle_{\mathfrak{g}}$ be a non-degenerate bi-invariant pairing on $\mathfrak{g}$, the Lie algebra of $G$, it extends complex bi-linearly to $\mathfrak{g}^{\mathbb{C}}$, the complexification of $\mathfrak{g}$. Let $*$ be the Cartan involution, in particular, $*^{2}=1$, then for $\xi, \eta \in \mathfrak{g}^{\mathbb{C}}$

$$
(\xi, \eta)_{\mathfrak{g}^{\mathbb{C}}}:=\left\langle\xi, \eta^{*}\right\rangle_{\mathfrak{g}^{\mathbb{C}}}
$$

defines a bi-invariant Hermitian inner product on $\mathfrak{g}^{\mathbb{C}}$. For any $z \in \mathcal{Z}$ the infinitesimal action

$$
\sigma_{z}: \mathfrak{g} \rightarrow T_{z} \mathcal{Z}
$$

is naturally extended to $\mathfrak{g}^{\mathbb{C}}$. Use the pairing $\langle\cdot, \cdot\rangle_{\mathfrak{g}}$ and the Kähler metric $\omega$ on $\mathcal{Z}$, we define

$$
Q_{z}:=\sigma_{z}^{*} \sigma_{z}: \mathfrak{g} \rightarrow \mathfrak{g}
$$


where $\sigma^{*}$ is the adjoint. The action of $G$ on $\mathcal{Z}$ is called Hamiltonian if there is an $A d$-equivariant moment map

$$
m: \mathcal{Z} \rightarrow \mathfrak{g}
$$

satisfying

$$
d\langle m(z), \xi\rangle_{\mathfrak{g}}=i_{\sigma_{z}(\xi)} \omega
$$

and the line bunde $L$ is called the pre-quantum line bundle.

\section{Definition 1.}

$$
\phi:=\|m\|^{2}=-\langle m, m\rangle_{\mathfrak{g}}: \mathcal{Z} \rightarrow \mathbb{R},
$$

The critical points of $\phi$ is called extremal points.

For $\forall w \in T_{z} Z$, we have

$$
\begin{aligned}
\langle\nabla \phi, w\rangle_{T_{z} Z} & =2\left\langle-\langle m(z), \nabla m(z)\rangle_{\mathfrak{g}}, w\right\rangle_{T_{z} \mathcal{Z}}=-2\left\langle m(z), \nabla_{w} m(z)\right\rangle_{\mathfrak{g}} \\
& =-2 \omega\left(\sigma_{z}(m(z)), w\right)=-2\left\langle I \sigma_{z}(m(z)), w\right\rangle_{T_{z} \mathcal{Z}}
\end{aligned}
$$

that is

$$
\nabla \phi(z)=-2 I \sigma_{z}(m(z))
$$

Definition 2. Let $z \in Z$ then

1. $z$ is called poly-stable with respect to $L$ if there is a $g \in G^{\mathbb{C}}$ such that $\phi(f \cdot z)=0$;

2. $z$ is called stable if $z$ is poly-stable and in addition the stabilizer $G_{z}$ is finite;

3. $z$ is called semistable if

$$
\inf _{g \in G^{\mathbb{C}}} \phi(g \cdot z)=0
$$

Let $\mathcal{Z}_{\text {ss }}$ denotes the open set of semi-stable points on $\mathcal{Z}$.

Kempf and Ness, Kirwan, Guillemin and Sternberg have shown that the above definition agree with the corresponding notion in geometric invariant theory(GIT). Here we give a criterion of determine the set $\mathcal{Z}_{s s}$. Let $\gamma_{0}$ be a lift of $z_{0} \in \mathcal{Z}$. Since $G^{\mathbb{C}}$ acts on $L$, we define $H: G^{\mathbb{C}} / G \rightarrow \mathbb{R}$ by

$$
H(g):=\log \left|g \cdot \gamma_{0}\right|
$$


and for $\xi \in \mathfrak{g}$ we define

$$
H_{\xi}(t):=H(\exp (\sqrt{-1} t \xi))
$$

then we have (see [DK] Section 6.5.2)

Proposition 3. 1. If $\gamma \in G^{\mathbb{C}} \cdot \gamma_{0}$, then $\gamma$ is a critical point of $H$ if and only if $m(\pi(\gamma))=0$, where $\pi: L \rightarrow X$.

2. Let $z_{t}:=\exp (\sqrt{-1} t \xi) \cdot z_{0}$. Then

$$
\begin{aligned}
& H_{\xi}^{\prime}(t)=-2\left\langle m\left(\exp (\sqrt{-1} t \xi) \cdot z_{0}\right), \xi\right\rangle_{\mathfrak{g}} \\
& H_{\xi}^{\prime \prime}(t)=2\left\|\sigma_{z_{t}}(\xi)\right\|^{2}
\end{aligned}
$$

Corollary 4. Let $\mathfrak{g}_{z}$ be the Lie algebra of the stabilizer $G_{z}$ then the following are equivalent

1. $z \in \mathcal{Z}$ is poly-stable.

2. For $\forall \xi \in \mathfrak{g}-\mathfrak{g}_{z}$ there is a unique minimum of $H_{\xi}(t)$

3. For $\forall \xi \in \mathfrak{g}-\mathfrak{g}_{z}$

$$
\lim _{t \rightarrow \infty} H_{\xi}^{\prime}(t)>0
$$

\subsection{Structures of $\mathfrak{g}_{z_{0}}$ •}

For $\xi \in \mathfrak{g}$ and $\eta \in \mathfrak{g}^{\mathbb{C}}$,

$$
\left\langle\xi, Q_{z}(\eta)\right\rangle_{\mathfrak{g}}=\left\langle\sigma_{z}(\xi), \sigma_{z}(\eta)\right\rangle_{T_{z} \mathcal{Z}}=\omega\left(\sigma_{z}(\xi), I \sigma_{z}(\eta)\right)=\left\langle\xi, d m \circ I \circ \sigma_{z}(\eta)\right\rangle_{\mathfrak{g}} .
$$

This together with the $A d$-invariance of $m$ imply the following:

Lemma 5. For $\xi, \eta \in \mathfrak{g}$,

$$
\begin{aligned}
\langle m(z),[\xi, \eta]\rangle_{\mathfrak{g}^{\mathbb{C}}} & =\langle[m(z), \xi], \eta\rangle_{\mathfrak{g}^{\mathbb{C}}} \\
& =\left\langle\sigma_{z}(\sqrt{-1} \xi), \sigma_{z}(\eta)\right\rangle_{T_{z} \mathcal{Z}}=\left\langle Q_{z}(\sqrt{-1} \xi), \eta\right\rangle_{\mathfrak{g}^{\mathbb{C}}}
\end{aligned}
$$

Proposition 6. Suppose $\xi \in \operatorname{ker}\left(\sigma_{z}\right)=\mathfrak{g}_{z}^{\mathbb{C}}$, the Lie algebra of $G_{z}^{\mathbb{C}}$. Then $f(g):=\left\langle m(g \cdot z), A d_{g} \xi\right\rangle_{\mathfrak{g}^{\mathbb{C}}}$ is constant along $G^{\mathbb{C}}$-orbit. In particular, $\overline{G^{\mathbb{C}} \cdot z} \cap m^{-1}(0) \neq \varnothing$ implies $\langle m(z), \xi\rangle_{\mathfrak{g}^{\mathbb{C}}}=0$. 
Proof. Suppose $\xi \in \mathfrak{g}^{\mathbb{C}}$, then for $\forall \eta \in \mathfrak{g}$, by $A d$-equivariance of moment map we have

$$
\left\langle m\left(e^{t \eta} \cdot z\right), A d_{e^{t \eta} \xi}\right\rangle_{\mathfrak{g}^{\mathbb{C}}}=\left\langle m(z), A d_{e^{-t \eta}} A d_{e^{t \eta}} \xi\right\rangle_{\mathfrak{g}^{\mathbb{C}}}=\langle m(z), \xi\rangle_{\mathfrak{g}^{\mathbb{C}}} .
$$

This shows that $f$ is constant along $G$-orbit. So we only need to deal with $\sqrt{-1} t \eta \in \mathfrak{g}^{\mathbb{C}}$ direction.

$$
\begin{aligned}
& \left.2 \frac{d}{d t}\right|_{t=0}\left\langle m\left(e^{\sqrt{-1} t \eta} \cdot z\right), A d_{e^{\sqrt{-1} t \eta}} \xi\right\rangle_{\mathfrak{g}^{\mathbb{C}}} \\
& =2\left\langle d m \circ I \circ \sigma_{z}(\eta), \xi\right\rangle_{\mathfrak{g}^{\mathbb{C}}}+2\langle m(z),[\sqrt{-1} \eta, \xi]\rangle_{\mathfrak{g}^{\mathbb{C}}} \\
& =\left\langle d m \circ I \circ \sigma_{z}(\eta), \xi+\xi^{*}+\xi-\xi^{*}\right\rangle_{\mathfrak{g}^{\mathbb{C}}}-\sqrt{-1}\left\langle[\eta, m(z)], \xi+\xi^{*}+\xi-\xi^{*}\right\rangle_{\mathfrak{g}^{\mathbb{C}}} \\
& =\left\langle\sigma_{z}^{*} \sigma_{z}(\eta), \xi-\xi^{*}\right\rangle_{\mathfrak{g}^{\mathbb{C}}}-\sqrt{-1}\left\langle\sigma_{z}^{*} \sigma_{z}(\eta), \sqrt{-1} \xi+\sqrt{-1} \xi^{*}\right\rangle_{\mathfrak{g}^{\mathbb{C}}} \\
& -\sqrt{-1}\left\langle[\eta, m(z)], \xi-\xi^{*}\right\rangle_{\mathfrak{g}^{\mathbb{C}}}-\left\langle[\eta, m(z)], \sqrt{-1} \xi+\sqrt{-1} \xi^{*}\right\rangle_{\mathfrak{g}^{\mathbb{C}}} \\
& =-\left\langle\sigma_{z}(\eta), \sigma_{z}\left(\xi^{*}\right)\right\rangle_{T_{z} Z}-\sqrt{-1}\left\langle\sigma_{z}(\eta), \sigma_{z}\left(\sqrt{-1} \xi^{*}\right)\right\rangle_{T_{z} Z} \\
& +\sqrt{-1}\left\langle\sigma_{z}^{*} \sigma_{z}(\sqrt{-1} \eta), \xi-\xi^{*}\right\rangle_{\mathfrak{g}^{\mathbb{C}}}+\left\langle\sigma_{z}^{*} \sigma_{z}(\sqrt{-1} \eta), \sqrt{-1} \xi+\sqrt{-1} \xi^{*}\right\rangle_{\mathfrak{g}^{\mathbb{C}}} \\
& =-\left\langle\sigma_{z}(\eta), \sigma_{z}\left(\xi^{*}\right)\right\rangle_{T_{z} Z}-\sqrt{-1}\left\langle\sigma_{z}(\eta), \sigma_{z}\left(\sqrt{-1} \xi^{*}\right)\right\rangle_{T_{z} Z} \\
& -\sqrt{-1}\left\langle\sigma_{z}(\sqrt{-1} \eta), \sigma_{z}\left(\xi^{*}\right)\right\rangle_{T_{z} Z}+\left\langle\sigma_{z}(\sqrt{-1} \eta), \sigma_{z}\left(\sqrt{-1} \xi^{*}\right)\right\rangle_{T_{z} Z} \\
& =-\left\langle\sigma_{z}(\eta), \sigma_{z}\left(\xi^{*}\right)\right\rangle_{T_{z} Z}-\sqrt{-1}\left\langle\sigma_{z}(\eta), \sigma_{z}\left(\sqrt{-1} \xi^{*}\right)\right\rangle_{T_{z} Z} \\
& +\sqrt{-1}\left\langle\sigma_{z}(\eta), \sigma_{z}\left(\sqrt{-1} \xi^{*}\right)\right\rangle_{T_{z} Z}+\left\langle\sigma_{z}(\eta), \sigma_{z}\left(\xi^{*}\right)\right\rangle_{T_{z} Z} \\
& =0
\end{aligned}
$$

Remark 7. Note that if $\xi$ lies in the reductive part of $\mathfrak{g}_{z}$ then $\langle m(z), \xi\rangle_{\mathfrak{g}^{\mathbb{C}}}$ is actually the weight of the $\mathbb{C}^{\times}$-action corresponding to $\xi$ on the fiber $L_{z}$. Clearly, it is constant along $G^{\mathbb{C}}$-orbit.

Corollary 8. Let $\xi, \eta \in \mathfrak{g}_{z}^{\mathbb{C}}$ then $\langle m(z),[\eta, \xi]\rangle_{\mathfrak{g}^{\mathbb{C}}}=0$. In particular, the map $\langle m(z), \cdot\rangle_{\mathfrak{g}^{\mathbb{C}}}: \mathfrak{g}_{z}^{\mathbb{C}} \rightarrow \mathbb{C}$ is a Lie algebra homomorphism.

Proof. Since

and this implies

$$
\left\langle m\left(e^{t \eta} \cdot z\right), A d_{e^{t \eta}} \xi\right\rangle_{\mathfrak{g}^{\mathbb{C}}}=\langle m(z), \xi\rangle_{\mathfrak{g}^{\mathbb{C}}}
$$

$$
0=\left.\frac{d}{d t}\right|_{t=0}\left\langle m\left(e^{t \eta} \cdot z\right), A d_{e^{t \eta}} \xi\right\rangle_{\mathfrak{g}^{\mathbb{C}}}=\left\langle d m \circ \sigma_{z}(\eta), \xi\right\rangle_{\mathfrak{g}^{\mathbb{C}}}+\langle m(z),[\eta, \xi]\rangle_{\mathfrak{g}^{\mathbb{C}}}
$$


The statement then follows from the assumption $\eta \in \mathfrak{g}_{z}^{\mathbb{C}}$.

Corollary 9. Let $z_{0} \in \mathcal{Z}$ and $z_{0} \in \overline{G^{\mathbb{C}} \cdot z}$. Suppose the stabilizer $\mathfrak{g}_{z_{0}}^{\mathbb{C}} \neq 0$ is simple and $\mathfrak{g}_{z}^{\mathbb{C}}=0$. Then $z$ is unstable.

Proof. By the assumption $z_{0} \in \overline{G^{\mathbb{C}} \cdot z}$, there is a $\xi \in \mathfrak{g}_{z_{0}}$ such that

$$
\lim _{t \rightarrow \infty} e^{-\sqrt{-1} t \xi} z=z_{0}
$$

Suppose $z$ is stable then it follows from Corollary 4

$$
2\langle m(z), \xi\rangle_{\mathfrak{g}}=\lim _{t \rightarrow \infty} H_{\xi}^{\prime}(t)>0
$$

On the other hand, Corollary 8 tells us $\left\langle m(z), \mathfrak{g}_{z_{0}}^{\mathbb{C}}\right\rangle_{\mathfrak{g}}=0$ since $\mathfrak{g}_{z_{0}}^{\mathbb{C}}$ is simple, this is a contradiction.

Theorem 10. Let $G$ be a compact Lie group acting on $(\mathcal{Z}, \omega)$ holomorphic isometrically . Suppose $z_{0}$ is a extremal point of $\phi$ then we have the following decomposition

$$
\mathfrak{g}_{z_{0}}^{\mathbb{C}}=\mathfrak{h}_{0} \oplus \bigcup_{\lambda>0} \mathfrak{h}_{\lambda}
$$

where

$$
\mathfrak{h}_{\lambda}:=\left\{\xi \in \mathfrak{g}_{z_{0}}^{\mathbb{C}} \mid\left[\sqrt{-1} m\left(z_{0}\right), \xi\right]=\lambda \cdot \xi\right\}
$$

and $\mathfrak{h}_{0}$ is the reductive part of $\mathfrak{g}_{z_{0}}^{\mathbb{C}}$. Moreover

$$
\left[\mathfrak{h}_{\lambda_{1}}, \mathfrak{h}_{\lambda_{2}}\right] \subset \mathfrak{h}_{\lambda_{1}+\lambda_{2}}
$$

and

$$
m\left(z_{0}\right) \in \mathcal{Z}\left(\mathfrak{h}_{0}\right),
$$

where $\mathcal{Z}\left(\mathfrak{h}_{0}\right)$ is the center of $\mathfrak{h}_{0}$.

Proof. Since $\mathfrak{g}_{z_{0}}^{\mathbb{C}}=\operatorname{ker}\left(\sigma_{z_{0}}\right) \subset \mathfrak{g}^{\mathbb{C}}$, we have decomposition

$$
\mathfrak{g}_{z_{0}}=\left(\mathfrak{g}_{z_{0}}^{\mathbb{C}} \cap \mathfrak{g}\right) \oplus \sqrt{-1}\left(\mathfrak{g}_{z_{0}}^{\mathbb{C}} \cap \mathfrak{g}\right) \oplus \mathfrak{n}_{z_{0}}
$$

where $\mathfrak{n}_{z}$ is the nilpotent radical. 
First, we claim that the action of $\left[\sqrt{-1} m\left(z_{0}\right), \cdot\right]$ on $g^{\mathbb{C}}$ is self adjoint. This is because that $(\xi, \eta):=\left\langle\xi, \eta^{*}\right\rangle_{\mathfrak{g}^{\mathbb{C}}}$ forms a Hermitian inner product on $\mathfrak{g}^{\mathbb{C}}$, where $*$ is the Cartan involution, and

$$
\begin{aligned}
\left(\left[\sqrt{-1} m\left(z_{0}\right), \xi\right], \eta\right) & =\left\langle\left[\sqrt{-1} m\left(z_{0}\right), \xi\right], \eta^{*}\right\rangle_{\mathfrak{g}^{\mathbb{C}}} \\
& =-\left\langle\xi,\left[\sqrt{-1} m\left(z_{0}\right), \eta^{*}\right]\right\rangle_{\mathfrak{g}^{\mathbb{C}}} \\
& =\left\langle\xi,\left[\left(-\sqrt{-1} m\left(z_{0}\right)\right)^{*}, \eta^{*}\right]\right\rangle_{\mathfrak{g}^{\mathbb{C}}} \\
& =\left(\xi,\left[\sqrt{-1} m\left(z_{0}\right), \eta\right]\right) .
\end{aligned}
$$

Secondly, since $\sqrt{-1} m\left(z_{0}\right)=\left(\sqrt{-1} m\left(z_{0}\right)\right)^{*}$,for $\forall \xi \in \mathfrak{g}_{z}$, if $\left[\sqrt{-1} m\left(z_{0}\right), \xi\right]=\lambda \xi$ then

$$
\left[\sqrt{-1} m\left(z_{0}\right), \xi^{*}\right]=\left[\left(\sqrt{-1} m\left(z_{0}\right)\right)^{*}, \xi^{*}\right]=-\left[\sqrt{-1} m\left(z_{0}\right), \xi\right]^{*}=-\lambda \xi^{*} .
$$

Since $\xi \in \mathfrak{g}_{z_{0}}^{\mathbb{C}}$ and $-(\sqrt{-1} \xi)^{*}+\sqrt{-1} \xi \in \mathfrak{g}$, this implies

$$
\begin{aligned}
2 \lambda\|\xi\|^{2} & =2 \lambda\left\langle\xi, \xi^{*}\right\rangle_{\mathfrak{g}^{\mathbb{C}}} \\
& =\lambda\left\langle\xi+\xi^{*}, \xi^{*}+\xi\right\rangle_{\mathfrak{g}^{\mathbb{C}}} \\
& =\left\langle\left[\sqrt{-1} m\left(z_{0}\right), \xi-\xi^{*}\right], \xi^{*}+\xi\right\rangle_{\mathfrak{g}^{\mathbb{C}}} \\
& =\left\langle\left[m\left(z_{0}\right), \xi-\xi^{*}\right], \sqrt{-1} \xi^{*}+\sqrt{-1} \xi\right\rangle_{\mathfrak{g}^{\mathbb{C}}} \\
& =\left\langle\left[m\left(z_{0}\right), \xi-\xi^{*}\right],-(\sqrt{-1} \xi)^{*}+\sqrt{-1} \xi\right\rangle_{\mathfrak{g}^{\mathbb{C}}}
\end{aligned}
$$

Now apply Lemma 5 with $\eta=-(\sqrt{-1} \xi)^{*}+\sqrt{-1} \xi$ we deduce

$$
\begin{aligned}
2 \lambda\|\xi\|^{2} & =\left\langle\left[m\left(z_{0}\right), \xi-\xi^{*}\right],-(\sqrt{-1} \xi)^{*}+\sqrt{-1} \xi\right\rangle_{\mathfrak{g}^{\mathbb{C}}} \\
& =\left\langle\sigma_{z_{0}}\left(\sqrt{-1} \xi-\sqrt{-1} \xi^{*}\right), \sigma_{z_{0}}\left(-(\sqrt{-1} \xi)^{*}+\sqrt{-1} \xi\right)\right\rangle_{T_{z_{0}} \mathcal{Z}} \\
& =\left\|\sigma_{z_{0}}\left(\xi^{*}\right)\right\|^{2}
\end{aligned}
$$

This implies that $\lambda \geq 0$. Moreover if $\lambda=0$ then we have $\sigma_{z_{0}}\left(\xi^{*}\right)=0$, and by our assumption $\sigma_{z_{0}}(\xi)=0$, so $\sigma_{z_{0}}\left(\xi-\xi^{*}\right)=0$. This implies that $\xi-\xi^{*} \in$ $\mathfrak{g}$ so we have $\xi \in\left(\mathfrak{g}_{z_{0}}^{\mathbb{C}} \cap \mathfrak{g}\right)^{\mathbb{C}}=\mathfrak{h}_{0}$ and $m\left(z_{0}\right) \in \mathcal{Z}\left(\mathfrak{h}_{0}\right)$. Clearly $\mathfrak{h}_{0}$ is the reductive part of $\mathfrak{g}_{z_{0}}$.

Finally, the relation

$$
\left[\mathfrak{h}_{\lambda_{1}}, \mathfrak{h}_{\lambda_{2}}\right] \subset \mathfrak{h}_{\lambda_{1}+\lambda_{2}} .
$$

easily follows from the Jacobi identity. 
Remark 11. If $\mathcal{Z}$ and $G$ both are of finite dimensional Proposition above is well known (see for instance $[K]$ ). Since the argument above is purely local, our approach also works for infinite dimensional $G$ as long as $\mathfrak{g}_{z_{0}}^{\mathbb{C}}$ is contained in a finite dimensional reductive Lie algebra.

Corollary 12. 1. If $m\left(z_{0}\right)=0$ then $\mathfrak{g}_{z_{0}}^{\mathbb{C}}$ is reductive.

2. If $z_{0}$ is a extremal point and for $\forall \xi \in \mathfrak{h}_{0},\left\langle m\left(z_{0}\right), \xi\right\rangle_{\mathfrak{g}^{\mathbb{C}}}=0$ then $m\left(z_{0}\right)=0$.

3. If $z_{0}$ is a extremal point, and $m\left(z_{0}\right) \neq 0$ then $\mathfrak{g}_{z_{0}}^{\mathbb{C}} \supset \mathfrak{u}(1)$.

4. Assume $G$ is finite dimensional, let $\xi \in \mathfrak{g}_{z}$ with $\langle m(z), \xi\rangle_{\mathfrak{g}^{\mathbb{C}}} \neq 0$ and $z_{0} \in \overline{G^{\mathbb{C}} \cdot z}$ be the extremal point of $\phi$. Then $\xi-\xi^{*} \in \mathfrak{g}_{z_{0}}$.

\section{Symplectic geometry of Chow and Hilbert scheme.}

\subsection{Stability of polarized manifold.}

In this subsection, let us recall some basic geometric invariant theory that is used in the construction of moduli space of polarized variety. Given a sufficiently large number $k, L^{k}$ induces a $k t h$-embedding $X \subset \mathbb{P}^{N_{k}}$, where $N_{k}=\operatorname{dim} H^{0}\left(X, L^{k}\right)-1$.

Definition 13. The degree $d:=k^{n} \int_{X} c_{1}^{n}(L)$ divisor

$$
\begin{aligned}
\text { Chow }\left(X, L^{k}\right): & =\left\{\Lambda \in \mathbb{G}\left(N_{k}-n, N_{k}+1\right) \mid \quad \Lambda \cap X \neq \emptyset\right\} \\
& \subset \mathbb{P} H^{0}\left(\mathbb{G}\left(N_{k}-n, N_{k}+1\right), \mathcal{O}_{\mathbb{G}}(d)\right)
\end{aligned}
$$

is called the $k$ th-Chow point of $X$, here $\mathbb{G}\left(N_{k}-n, N_{k}+1\right)$ is the Grassmanian of $N_{k}-n$ dimensional linear subspace in $\mathbb{C}^{N_{k}+1}$. We define

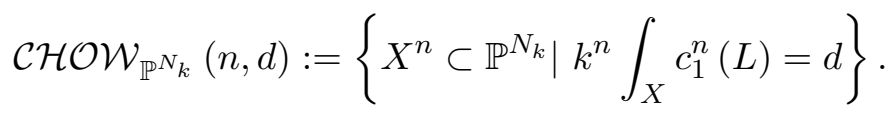

Clearly we have

$$
\mathcal{C H O} \mathcal{W}_{\mathbb{P}^{N_{k}}}(n, d) \subset \mathbb{P} H^{0}\left(\mathbb{G}\left(N_{k}-n, N_{k}+1\right), \mathcal{O}_{\mathbb{G}}(d)\right) .
$$

Definition 14. We define the kth-Hilbert point of $X$ to be

$$
\operatorname{Hilb}\left(X, L^{k}\right):=\mathcal{I}_{X} \in \mathcal{H} \mathcal{I} \mathcal{L B}_{\mathbb{P}^{N_{k}}}(\chi)
$$


where

$$
\begin{aligned}
& \mathcal{H} \mathcal{I} \mathcal{B}_{\mathbb{P}^{N_{k}}}(\chi) \\
= & \left\{\mathcal{I}_{X} \subset \mathcal{O}_{\mathbb{P}^{N_{k}}} \mid H^{0}\left(\mathbb{P}^{N_{k}}, \mathcal{I}_{X} \otimes_{\mathcal{O}_{\mathbb{P}^{N}}} \mathcal{O}_{\mathbb{P}^{N_{k}}}(m)\right)=\chi(m) \text { for } m>>1\right\}
\end{aligned}
$$

is the Hilbert scheme of $\mathbb{P}_{k}^{N}$ with Hilbert polynomial $\chi$.

It follows from the exact sequence

$$
0 \longrightarrow \mathcal{I}_{X}(m) \longrightarrow \mathcal{O}_{\mathbb{P}^{N_{k}}}(m) \longrightarrow \mathcal{O}_{X}(m) \longrightarrow 0
$$

that

$$
\mathcal{H} \mathcal{I} \mathcal{B}_{\mathbb{P}^{N_{k}}}(\chi) \subset \mathbb{G}\left(\chi(m), d_{k, m}\right) \stackrel{\text { Plücker }}{\longrightarrow} \mathbb{P}^{K-1}
$$

for $m>>1$, where

$$
K=\left(\begin{array}{c}
d_{k, m} \\
\chi(m)
\end{array}\right) \text { and } d_{k, m}=\operatorname{dim}_{\mathbb{C}} H^{0}\left(X, L^{k m}\right) .
$$

Definition 15. 1. We call $(X, L)$ Chow poly-stable for the kth-embedding $X \subset \mathbb{P}^{N_{k}}$ if $\operatorname{Chow}\left(X, L^{k}\right) \in \mathcal{C H O W}_{\mathbb{P}^{N}}(n, d)$ is poly-stable with respect to the natural $S L\left(N_{k}+1\right)$ action on $\left(\mathcal{C H O W}_{\mathbb{P}^{N_{k}}}(n, d), \mathcal{O}_{\mathcal{C H O W}_{\mathbb{P}^{N}}(n, d)}(1)\right)$. And we call $(X, L)$ is asymptotic Chow poly-stable if $(X, L)$ is Chow poly-stable for $k>>1$.

2. We call $(X, L)$ Hilbert poly-stable for the kth-embedding if $\operatorname{Hilb}\left(X, L^{k}\right) \in \mathcal{H} \mathcal{I} \mathcal{L B}_{\mathbb{P}^{N_{k}}}(\chi)$ is poly-stable with respect to the natural $S L\left(N_{k}+1\right)$ action on $\left(\mathcal{H} \mathcal{I} \mathcal{L} \mathcal{B}_{\mathbb{P}^{N_{k}}}(\chi), \mathcal{O}_{\mathcal{H} \mathcal{I} \mathcal{L} \mathcal{B}_{\mathbb{P}^{N}}}(\chi)(1)\right)$.

In particular we have the following relations between these two notions of stability (c.f. $[\mathrm{G}]$ and $[\mathrm{Mum}]$ )

Chow stable $\Longrightarrow$ Hilbert stable $\Longrightarrow$ Hilbert semi-stable $\Longrightarrow$ Chow semi-stable.

For the subsequent two sections we will apply the general theory of symplectic quotient established in Section 2 to both Chow and Hilbert scheme. Notice that in general Chow and Hilbert scheme both are NOT smooth, but for our purpose what we really care about is only a single $S L(N+1)$-orbit which is smooth, so the Section 2 is applicable to our situation. 


\subsection{Chow scheme.}

Since

$$
\mathcal{C H O} \mathcal{W}_{\mathbb{P}^{N}}(n, d) \subset \mathbb{P} H^{0}\left(\mathbb{G}\left(N_{k}-n, N_{k}+1\right), \mathcal{O}_{\mathbb{G}}(d)\right),
$$

it inherits a natural symplectic form from the Fubini-Study Kähler form on $\mathbb{P} H^{0}\left(\mathbb{G}\left(N_{k}-n, N_{k}+1\right), \mathcal{O}_{\mathbb{G}}(d)\right)$. Following [W], we may take another natural symplectic form on $\mathcal{C H O W}_{\mathbb{P}^{N_{k}}}(n, d)$ by considering the following diagram

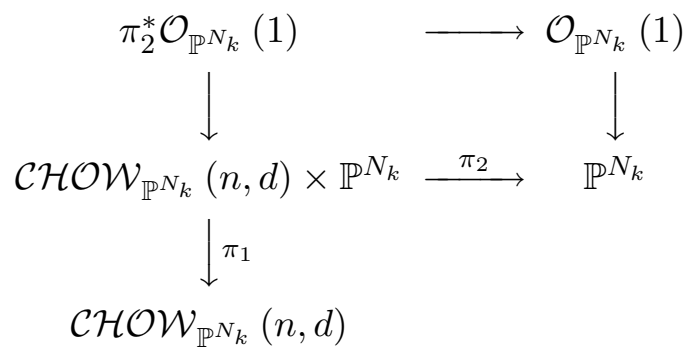

and let

$$
\mathcal{C H O W}_{\mathbb{P}^{N_{k}}}(n, d) \times \mathbb{P}^{N_{k}} \supset \Sigma:=\{(f, f(x)) \mid x \in X\}
$$

be the universal family then we define the symplectic form $\Omega$ on $\mathcal{C H O} \mathcal{W}_{\mathbb{P}^{N_{k}}}(n, d)$ by

$$
\Omega:=\pi_{1 *}\left(\frac{\left(\pi_{2}^{*} \omega_{F S}\right)^{n+1}}{(n+1) !} \cap[\Sigma]\right)
$$

Remark 16. In [Z], S.W. Zhang identified the pre-quantum line bundle for $\Omega$ with the Deligne-Pairing.

Now $S U\left(N_{k}+1\right)$ acts naturally on $\left(\mathcal{C H O W}_{\mathbb{P}^{N_{k}}}(n, d), \Omega\right)$ and the moment map is given by the following

\section{Proposition 17.}

$$
\begin{aligned}
\mu_{\Omega}\left(\operatorname{Chow}\left(X, L^{k}\right)\right) & =\int_{X} \mu_{F S} \frac{\omega_{F S}^{n}}{n !} \\
& =\frac{1}{2 \pi i} \int_{X}\left(\frac{z z^{*}}{|z|^{2}}-\frac{1}{N_{k}+1}\right) \frac{\omega_{F S}^{n}}{n !} \in \mathfrak{s u}\left(N_{k}+1\right),
\end{aligned}
$$

where $\mu_{F S}$ is the moment map of $\left(\mathbb{P}^{N_{k}}, \omega_{F S}\right)$ with respect to $S U\left(N_{k}+1\right)$ action. 
Proof. Let $\xi \in \mathfrak{s u}\left(N_{k}+1\right)$ and $X_{\xi} \in H^{0}\left(\mathbb{P}^{N_{k}}, T \mathbb{P}^{N_{k}}\right)$ be the induced holomorphic vector field on $\mathbb{P}^{N_{k}}$. If we identify $\operatorname{TCHOW}_{\text {Chow }(X)}$ with $H^{0}\left(X,\left.T \mathbb{P}^{N_{k}}\right|_{X}\right)$ then for $\forall Y \in \Gamma\left(X,\left.T \mathbb{P}^{N_{k}}\right|_{X}\right)$

$$
\begin{aligned}
& \Omega\left(\left.X_{\xi}\right|_{\text {Chow }\left(X, L^{k}\right)}, Y\right)
\end{aligned}
$$

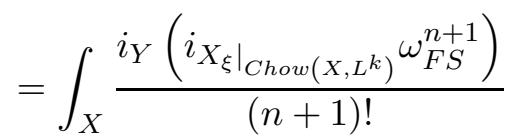

$$
\begin{aligned}
& =\int_{X} \frac{i_{Y}\left(i_{\left.X_{\xi}\right|_{\text {Chow }\left(X, L^{k}\right)}} \omega_{F S} \wedge \omega_{F S}^{n}\right)}{n !}
\end{aligned}
$$

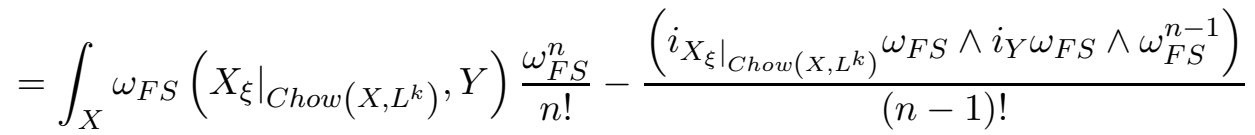

$$
\begin{aligned}
& =\int_{X} \operatorname{Tr}\left(d \mu_{F S}(Y) \cdot \xi\right) \frac{\omega_{F S}^{n}}{n !}-\int_{X} \bar{\partial} \mu_{F S}(\xi) \wedge \partial \mu_{F S}(Y) \frac{\omega_{F S}^{n-1}}{(n-1) !} .
\end{aligned}
$$

On the other hand

$$
\begin{aligned}
& \left\langle d \mu_{\Omega}(f)(Y), \xi\right\rangle \\
& =\left.\frac{d}{d t}\right|_{t=0}\left\langle\int_{X}\left(\frac{z z^{*}}{|z|^{2}}-\frac{1}{N_{k}+1}\right) \frac{f^{*} \omega_{F S}^{n}}{n !}, \xi\right\rangle \\
& =\int_{X} \operatorname{Tr}\left(d \mu_{F S}(Y) \cdot \xi\right) \frac{\omega_{F S}^{n}}{n !}+\int_{X} \operatorname{Tr}\left(\mu_{F S} \cdot \xi\right) \bar{\partial} \partial \mu_{F S}(Y) \frac{\omega_{F S}^{n-1}}{(n-1) !} \\
& =\int_{X} \operatorname{Tr}\left(d \mu_{F S}(Y) \cdot \xi\right) \frac{\omega_{F S}^{n}}{n !}-\int_{X} \bar{\partial} \mu_{F S}(\xi) \wedge \partial \mu_{F S}(Y) \frac{\omega_{F S}^{n-1}}{(n-1) !} .
\end{aligned}
$$

And clearly $\mu_{\Omega}$ is $A d$-equivariant because it is the integral of $A d$-equivariant $\mu_{F S}$.

Definition 18. The $k t h$-embedding of $X \subset \mathbb{P}^{N_{k}}$ induced by $L^{k}$ is called balanced if there is a $g \in S L\left(N_{k}+1\right)$, such that

$$
\mu_{\Omega}\left(\operatorname{Chow}\left(g \cdot X, L^{k}\right)\right)=\int_{g \cdot X} \mu_{F S} \frac{\omega_{F S}^{n}}{n !}=0
$$

Let $\mathfrak{a u t}(X, L) \subset \mathfrak{s l}(N+1)$ be the stabilizer of Chow $(X, L) \in$

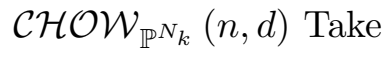


Definition 19. Let Chow $(X) \in \mathcal{C H O W}_{\mathbb{P}^{N_{k}}}(n, d)$ be the Chow point corresponding to the projective embedding $X \subset \mathbb{P}^{N_{k}}$, then for

$$
\forall \xi \in \mathfrak{a u t}(X, L) \subset \mathfrak{s l}(N+1)
$$

we define

$$
f_{X, k}(\xi):=\left\langle\mu_{\Omega}\left(\operatorname{Chow}_{k}(X)\right), \xi\right\rangle_{\mathfrak{s l}}
$$

and we say $X \subset \mathbf{P}^{N_{k}}$ can be balanced provided there is a $g \in S L\left(N_{k}+1\right)$ such that $\mu_{\Omega}\left(\right.$ Chow $\left._{k}(g \cdot X)\right)=0$.

It follows from Corollary 4, Propostion 6 and Theorem 10.

Proposition 20. 1. $f_{X, k}: \mathfrak{a u t}(X, L) \rightarrow \mathbb{C}$ is a Lie algebra homomorphism and is independent on the choice of basis of the projective embedding;

2. Chow $\left(X, L^{k}\right)$ is stable if and only if for any $\xi \in \mathfrak{s u}\left(N_{k}+1\right)$ we have

$$
\begin{aligned}
& \lim _{t \rightarrow \infty}\left\langle\mu_{\Omega}\left(e^{-\sqrt{-1} t \xi} \cdot \operatorname{Chow}\left(X, L^{k}\right)\right), \xi\right\rangle_{\mathfrak{s u}} \\
& =\lim _{t \rightarrow \infty}\left\langle\mu_{\Omega}\left(\operatorname{Chow}\left(e^{-\sqrt{-1} t \xi} \cdot X, L^{k}\right)\right), \xi\right\rangle_{\mathfrak{s u}}>0 ;
\end{aligned}
$$

3. If Chow $\left(X, L^{k}\right)$ is semi-stable then $f_{X, k}=0$. In particular, $f_{X, k}$ is the finite dimensional analogue of Futaki invariant.

Remark 21. In fact, it can be shown that $f_{X, k}$ is actually only depend on the Kähler class.

By applying a trick of Mabuchi $[\mathrm{M}]$ we have the following proposition.

Proposition 22. Suppose $\mathfrak{a} \mathfrak{u t}(X, L) \ni \xi$ is a nilpotent element. Then

$$
f_{X, k}(\xi)=0 .
$$

Proof. Since we learn from Proposition 6 that $f_{X, k}$ is independent of the projective embedding, we may assume that $\xi$ is already of Jordan block form, that is

$$
\xi=\left[\begin{array}{cccc}
0 & e_{1} & & \\
& \ddots & \ddots & \\
& & \ddots & e_{N_{k}-1} \\
& & & 0
\end{array}\right],
$$


where $e_{i}= \pm 1$. Now let

$$
S L\left(N_{k}+1\right) \ni g=\varepsilon^{-\frac{N_{k}\left(N_{k}+1\right)}{2}} \operatorname{diag}\left[\varepsilon^{N_{k}}, \varepsilon^{N_{k}-1}, \cdots, \varepsilon, 1\right]
$$

then we have

$$
\begin{aligned}
f_{X, k}(\xi) & =\left\langle\mu_{\Omega}\left(\operatorname{Chow}_{k}(X)\right), \xi\right\rangle_{\mathfrak{s l}} \\
& =\left\langle\mu_{\Omega}\left(\text { Chow }_{k}(g \cdot X)\right), A d_{g} \xi\right\rangle_{\mathfrak{s l}} \\
& =\frac{1}{2 \pi i} \int_{X} \operatorname{Tr}\left[\left(\frac{g \cdot z z^{*} \cdot g^{*}}{|g \cdot z|^{2}}-\frac{1}{N_{k}+1}\right)\left(g \cdot \xi \cdot g^{-1}\right)\right] \frac{\left(g^{*} \omega_{F S}\right)^{n}}{n !} \\
& =\frac{1}{2 \pi i} \int_{X} \frac{\operatorname{Tr}\left[z^{*} g^{*} g \xi z\right]}{|g \cdot z|^{2}} \frac{\left(g^{*} \omega_{F S}\right)^{n}}{n !} ;
\end{aligned}
$$

Notice

$$
\begin{aligned}
\left|\frac{\operatorname{Tr}\left[z^{*} g^{*} g \xi z\right]}{|g \cdot z|^{2}}\right| & \leq \varepsilon \frac{\left|\sum_{i=0}^{N_{k}-1} \varepsilon^{N_{k}-i} \bar{z}_{i} \varepsilon^{N_{k}-i-1} e_{i+1} z_{i+1}\right|}{\sum_{i=0}^{N_{k}}\left|z_{i}\right|^{2} \varepsilon^{2\left(N_{k}-i\right)}} \\
& \leq \varepsilon
\end{aligned}
$$

the last step follows from Cauchy-Schwartz inequality. Now we let $\varepsilon \rightarrow 0$, this implies $f_{X, k}(\xi)=0$.

Remark 23. Note Theorem 10 implies that $f_{X, k}$ enjoys all the properties possessed by Futaki invariant.

In order to find a geometric way to evaluate Chow weight we need the following two basic Lemmas.

Lemma 24. (Proposition I.7.4 in [HA]) Let $M$ be a finitely generated graded module over $S:=\mathbb{C}\left[z_{0}, \cdots, z_{N}\right]$. Then there exists a filtration $0=M^{0} \subset M^{1} \subset \cdots \subset M^{r}=M$ by graded submodules, such that for each $i, M^{i} / M^{i-1} \simeq\left(S / \mathfrak{p}_{i}\right)\left(l_{i}\right)$, the twisted module (i.e. $\left.\left(S / \mathfrak{p}_{i}\right)\left(l_{i}\right)_{d}:=\left(S / \mathfrak{p}_{i}\right)_{d+l_{i}}\right)$, where $\mathfrak{p}_{i}$ is a homogenous prime ideal of $S$, and $l_{i} \in \mathbb{Z}$. Moreover, we have:

1. if $\mathfrak{p}$ is a homogenous prime ideal of $S$, then

$$
\mathfrak{p} \supseteq \operatorname{Ann}(M):=\{s \in S \mid s \cdot M=0\} \Leftrightarrow \mathfrak{p} \supseteq \mathfrak{p}_{i}
$$

for some i. In particular, the minimal elements of the set $\left\{\mathfrak{p}_{1}, \cdots, \mathfrak{p}_{r}\right\}$ are just the minimal primes of $M$. 
2. for each minimal prime of $M$, the number of times which $\mathfrak{p}$ occurs in the set $\left\{\mathfrak{p}_{1}, \cdots, \mathfrak{p}_{r}\right\}$ is equal to $\mu_{\mathfrak{p}}(M)$, the length of $M_{\mathfrak{p}}$ over the local ring $S_{\mathfrak{p}}$.And $\mu_{\mathfrak{p}}(M)$ is called the multiplicity of $M$ at $\mathfrak{p}$.

To state the second one, let $\xi=\operatorname{diag}\left[\mu_{0}, \cdots, \mu_{N}\right] \in \mathfrak{s l}(N+1)$ and $X \subset$ $\mathbb{P}^{N}$ be a $e^{\xi t}$-invariant $n$-dimensional closed subscheme. $\xi$ induces a natural action $\wedge^{\text {top }} H^{0}\left(\mathbb{P}^{N}, \mathcal{O}_{\mathbb{P}^{N}}(k) / \mathcal{I}_{X} \otimes \mathcal{O}_{\mathbb{P}^{N}}(k)\right)$, where $\mathcal{I}_{X}$ is the ideal sheaf of $X$ and the weight polynomial is given by

$$
w_{X}(\xi)=w_{X, 0}(\xi) \frac{k^{n+1}}{(n+1) !}+w_{X, 1}(\xi) \frac{k^{n}}{n !}+\cdots+w_{X, n+1}(\xi) .
$$

We call $w_{X}(\xi)$ the $\xi$-weight of $X$.

Lemma 25. Suppose $X \subset \mathbb{P}^{N}$ be a $\xi$-invariant $n$-dimensional closed subscheme with irreducible components $X_{1}, \cdots, X_{s}$, each component $X_{i}$ has multiplicity $a_{i}$ in $X$. Then

$$
w_{X, 0}(\xi)=\sum_{i=1}^{s} a_{i} w_{X_{i}, 0}(\xi)
$$

Proof. Let $M:=\oplus_{k=0}^{\infty} H^{0}\left(\mathbb{P}^{N}, \mathcal{O}_{\mathbb{P}^{N}}(k) / \mathcal{I}_{X}(k)\right)$. Then $M$ is a graded $S$-module, in particular $X=\operatorname{Proj}(M)$. From Lemma 24, we know that $M$ has a filtration $0=M^{0} \subset M^{1} \subset \cdots \subset M^{q}=M$, whose quotients $M^{i} / M^{i-1}$ are of the form $\left(S / \mathfrak{q}_{i}\right)\left(l_{i}\right)$, where $\mathfrak{q}_{i}$ is a homogenous prime ideal. If zero locus $Z\left(\mathfrak{q}_{i}\right)$ is a projective variety of dimension $r_{i}$ then

$$
w_{Z\left(\mathfrak{q}_{i}\right)}(\xi)=w_{Z\left(\mathfrak{q}_{i}\right), 0}(\xi) \frac{k^{r_{i}+1}}{\left(r_{i}+1\right) !}+w_{Z\left(\mathfrak{q}_{i}\right), 1}(\xi) \frac{k^{r_{i}}}{r_{i} !}+\cdots
$$

Note that the shift $l_{i}$ does not affect the leading coefficient in above. Since we are interested only in the leading coefficient, we can ignore those $w_{Z\left(\mathfrak{q}_{i}\right)}(\xi)$ of degree $<n+1$. We are left with those $w_{Z\left(\mathfrak{q}_{i}\right)}(\xi)$ where $\mathfrak{q}_{i}$ is a minimal prime of $M$, namely one of the primes $\mathfrak{p}_{1}, \cdots, \mathfrak{p}_{q}$ corresponds to $X_{i}$, and each one of these occurs $\mu_{\mathfrak{p}_{i}}(M)$ times. Since the weight polynomial is an additive function, this implies

$$
w_{M}=\sum_{i=1}^{q} w_{M^{i} / M^{i-1}} .
$$

In particular, we get $w_{X, 0}(\xi)=\sum_{i=1}^{s} a_{i} w_{X_{i}, 0}(\xi)$. 
For $\forall \xi \in \mathfrak{s u}(N+1)-\mathfrak{a u t}(X, L)$, we if let $X_{\infty}$ denote flat limit

$$
\lim _{t \rightarrow \infty} e^{\sqrt{-1} t \xi} \cdot X
$$

then $X_{\infty}$ is $\xi$-invariant and we have the following:

\section{Theorem 26.}

$$
\frac{w_{X_{\infty}, 0}(\xi)}{n+1}=\lim _{t \rightarrow \infty}\left\langle\mu_{\Omega}\left(e^{\sqrt{-1} t \xi} \operatorname{Chow}(X, L)\right), \xi\right\rangle_{\mathfrak{s u}}
$$

Proof. Suppose $Y_{1}, \cdots, Y_{s}$ are the irreducible components of $X_{\infty}$ and $a_{i}$ is the multiplicity of $Y_{i}$ in $X_{\infty}$. Then as a algebraic cycle, we have

$$
\left[X_{\infty}\right]=\sum_{i=1}^{s} a_{i}\left[Y_{i}\right] .
$$

By the continuity of integral we have

$$
\begin{aligned}
& \lim _{t \rightarrow \infty}\left\langle\mu_{\Omega}\left(e^{\sqrt{-1} t \xi} \cdot \operatorname{Chow}(X, L)\right), \xi\right\rangle_{\mathfrak{s u}} \\
& =\lim _{t \rightarrow \infty}\left\langle\mu_{\Omega}\left(\operatorname{Chow}\left(e^{\sqrt{-1} t \xi} \cdot X, L\right)\right), \xi\right\rangle_{\mathfrak{s u}} \\
& =\lim _{t \rightarrow \infty} \int_{e^{\sqrt{-1} t \xi} \cdot X} \mu_{F S}(\xi) \omega_{F S}^{n} \\
& =\sum_{i=1}^{s} a_{i} \int_{Y_{i}} \mu_{F S}(\xi) \omega_{F S}^{n}
\end{aligned}
$$

Step 1. Suppose $Y \subset \mathbb{P}^{N}$ is a linear subspace invariant under the action of $e^{\sqrt{-1} t \xi}$. Without loss of generality we may assume

$$
\xi:=\operatorname{diag}\left[i \xi_{0}, \cdots, i \xi_{N}\right] \in \mathfrak{s u}(N+1)
$$

and

$$
Y:=\left\{z_{n+1}=\cdots=z_{N}=0\right\},
$$

then we have

$$
\begin{aligned}
\int_{Y} \mu_{F S}(\xi) \omega_{F S}^{n} & =\int_{\mathbb{P}^{n}} \mu_{F S}(\xi) \omega_{F S}^{n} \\
& =\int_{\mathbb{P}^{n}} \frac{\sum_{i=0}^{n} \xi_{i}\left|z_{i}\right|^{2}}{\sum_{i=0}^{n}\left|z_{i}\right|^{2}} \omega_{F S}^{n} \\
& =\frac{\sum_{i=0}^{n} \xi_{i}}{n+1} .
\end{aligned}
$$


Also $w_{Y, 0}(\xi)=\sum_{i=0}^{n} \xi_{i}$, since $[Y]=\left[e_{0} \wedge \cdots \wedge e_{n}\right] \in \mathbb{G}(N-n, N+1)$. We thus obtain

$$
\frac{w_{Y, 0}(\xi)}{n+1}=\int_{Y} \mu_{F S}(\xi) \omega_{F S}^{n}=\left\langle\mu_{\Omega}(\operatorname{Chow}(Y, L)), \xi\right\rangle
$$

Step 2. Suppose $Y \subset \mathbb{P}^{N}$ is an irreducible closed sub-scheme which is $e^{t \xi}$-invariant. We may take a 1 -parameter subgroup $\lambda(t): \mathbb{C}^{\times} \longrightarrow$ $S L(N+1)$ such that $\lambda(t)$ commute with $e^{t \xi}$ and

$$
Y_{\infty}:=\lim _{t \rightarrow \infty} \lambda(t) \cdot Y=\sum_{j=1}^{l} b_{j} L_{i}
$$

with $L_{i}^{\prime} s$ being $\lambda$-invariant linear subspaces. Since $\lambda(t) \cdot Y$ form a flat family over $\mathbb{C}$, this implies $w_{Y, 0}(\xi)=w_{Y_{\infty}, 0}(\xi)$. Now it follows from Lemma 25 that

$$
\frac{w_{Y_{\infty}, 0}(\xi)}{n+1}=\frac{1}{n+1} \sum_{j=1}^{l} b_{i} \cdot w_{L_{i}, 0}(\xi)=\sum_{j=1}^{l} b_{i} \cdot\left\langle\mu_{\Omega}\left(\operatorname{Chow}\left(L_{i}, L\right)\right), \xi\right\rangle .
$$

On the other hand, since $\xi$ lies in the stabilizer of Chow $(Y, L)$ and $\lambda(t) \in$ $S L(N+1)$, we can deduce from Proposition 6 that

$$
\begin{aligned}
\left\langle\mu_{\Omega}(\operatorname{Chow}(Y, L)), \xi\right\rangle & =\left\langle\mu_{\Omega}(\operatorname{Chow}(\lambda(t) \cdot Y, L)), \xi\right\rangle \\
& =\sum_{j=1}^{l} b_{i} \cdot\left\langle\mu_{\Omega}\left(\operatorname{Chow}\left(L_{j}, L\right)\right), \xi\right\rangle \\
& =\frac{w_{Y_{\infty}, 0}(\xi)}{n+1}=\frac{w_{Y, 0}(\xi)}{n+1}
\end{aligned}
$$

Step 3. By Lemma 25

$$
w_{X_{\infty}, 0}(\xi)=\sum_{j=1}^{s} a_{j} w_{Y_{j}, 0}(\xi) .
$$

Now we put everything together

$$
\begin{aligned}
\frac{w_{X_{\infty}, 0}(\xi)}{n+1} & =\frac{1}{n+1} \sum_{j=1}^{s} a_{i} \cdot w_{Y_{j}, 0}(\xi) \\
& =\sum_{j=1}^{s} \alpha_{j}\left\langle\mu_{\Omega}\left(\operatorname{Chow}\left(Y_{j}, L\right)\right), \xi\right\rangle \\
& =\lim _{t \rightarrow \infty}\left\langle\mu_{\Omega}\left(e^{\sqrt{-1} t \xi} \operatorname{Chow}(X, L)\right), \xi\right\rangle_{\mathfrak{s u}} .
\end{aligned}
$$


Now $\xi$ lies in the stabilizer of $\operatorname{Chow}\left(X_{\infty}, L\right)$, the $\xi$-weight of Chow $(X, L)$ is defined to be the weight of the $\mathbb{C}^{\times}$-action induced by $\xi$ on $\left.\mathcal{O}_{\mathcal{C H O W}_{\text {PN }}(n, d)}(1)\right|_{\text {Chow }\left(X_{\infty}, L\right)}$. Then we have the following easy consequence originally due to Zhang, an alternative proof was gaven by Paul.

Corollary 27. ([Z] $[\mathrm{Luo}][\mathrm{P}])$ Let $(X, L)$ be a projective variety polarized by a very ample line bundle $L$. Then $C h o w(X, L)$ is poly-stable if and only if the projective embedding $X \stackrel{L}{\longrightarrow} \mathbb{P}^{N}$ induced by $L$ can be balanced.

Proof. It follows from Proposition 2.11 of [Mum]

$$
\xi \text { - weight of } \operatorname{Chow}(X, L)=w_{X_{\infty}, 0}(\xi) .
$$

By Hilbert-Mumford criterion $[\mathrm{MFK}]$, being Chow poly-stable means $\xi-$ weight of $C h o w(X, L)<0$ for $\forall \xi \in \mathfrak{s u}(N+1)-\mathfrak{a u t}(X, L)$. From the Theorem above, this is equivalent to

$\lim _{t \rightarrow \infty}-\left\langle\mu_{\Omega}\left(e^{\sqrt{-1} t \xi} \operatorname{Chow}(X, L)\right), \xi\right\rangle_{\mathfrak{s u}}>0$ for $\forall \xi \in \mathfrak{s u}(N+1)-\mathfrak{a u t}(X, L)$.

The statement then follows from Corollary 4 and Proposition 17.

Remark 28. Notice that the above Theorem tells us that the open set of poly-stable points on Chow scheme are the same with respect two different polarisation. For vector bundle case, this is corresponding to Simpson's construction versus Gieseker's construction(see [HL]).

\subsection{Hilbert scheme.}

For Hilbert scheme, if we let $m>>1$ then from the construction of Hilbert scheme it is clear that the pre-quantum line bundle is given by

$$
\operatorname{det} H^{0}\left(X, L^{k m}\right) \simeq \mathcal{O}_{\mathbb{P}^{K-1}}(1)
$$

where $K$ and $d_{k, m}$ are as in Section 4.1. And the action of $S U\left(N_{k}+1\right)$ on $\mathbb{P}^{N}$ naturally induces a Hamiltonian action on $\mathcal{H} \mathcal{I} \mathcal{L B}_{\mathbb{P}^{N_{k}}}(\chi)$. Now suppose $S L\left(N_{k}+1\right) \supset \mathfrak{a u t}(X, L) \neq\{0\}$ then the Hilbert point version of Proposition 20 is

Proposition 29. Suppose the $k t h$-Hilbert point

$$
\operatorname{Hilb}\left(X, L^{k}\right) \in \mathcal{H} \mathcal{I} \mathcal{L} \mathcal{B}_{\mathbb{P}^{N_{k}}}(\chi)
$$


is semi-stable. Then for any $\xi \in \mathfrak{a u t}(X, L)$

$$
\left\langle\mu_{F S}\left(\operatorname{Hilb}\left(X, L^{k}\right)\right), \xi\right\rangle_{\mathfrak{s u}}=0
$$

for $m>>1$, where $\mu_{F S}$ is the restriction of the standard moment map on $\mathbb{P}^{K-1}$ to $\mathcal{H} \mathcal{I} \mathcal{L B}_{\mathbb{P}^{N}}(\chi)$.

Let us consider an $S^{1}$ - action on $(X, L)$, so we can fix a $S^{1}$-invariant Hermitian metric $h$ on $L$ and let $\omega=\frac{i}{2 \pi} \operatorname{Ric}(h)$ be the Kähler form on $X$. Suppose that the action is Hamiltonian so it lifts to an action on $L \rightarrow X$ thus acts on $H^{0}\left(X, L^{k}\right)$ for $\forall k$. So for each $k$ we can get a homomorphism

$$
\rho_{k}: S^{1} \rightarrow \operatorname{Aut}_{0}(X) \subset S L\left(N_{k}+1\right) .
$$

If we let $w_{k, m}$ denote the weight of the $S^{1}$-action on $\wedge^{t o p} H^{0}\left(X, L^{k m}\right)$ for $\forall m$ induced by $\rho_{k}$ then we have

Corollary 30. Suppose that $(X, L, \omega)$ has a Hamiltonian $S^{1}$-action and the $k t h$-Hilbert point Hilb $\left(X, L^{k}\right)$ is semi-stable. Then $w_{k, m}=0$, for $m>>1$.

Now

$$
w_{k, m}=A_{k} m^{n+1}+B_{k} m^{n}+O\left(m^{n-1}\right)
$$

and equivariant Riemann-Roch theorem tells us that

$$
\begin{aligned}
A_{k} & =\int_{X}\left\langle\mu_{\omega_{F S}, k}, \xi\right\rangle_{\mathfrak{s u}} \frac{\omega_{F S}^{n}}{n !} \\
B_{k} & =\int_{X}\left\langle\mu_{\omega_{F S}, k}, \xi\right\rangle_{\mathfrak{s u}} \frac{c_{1}(X) \wedge \omega_{F S}^{n-1}}{2(n-1) !}
\end{aligned}
$$

The dependence of $A_{k}$ on $k$ will be made more explicit in next section. In particular, we have

Corollary 31. Suppose that the kth-Hilbert point Hilb $\left(X, L^{k}\right)$ is semistable then $B_{k} \equiv 0$.

Remark 32. Notice that $A=f_{X, k}$, and if $A=0$, then $B=F_{X}$ is the classical Futaki character(see the definition in the next section).

\section{Futaki invariant.}

In this section we compare the infinite and finite dimensional description of the classical Futaki invariant $F_{X}: \mathfrak{a u t}(X) \longrightarrow \mathbb{C}$. 


\subsection{Infinite dimensional picture.}

In this subsection we recall Fujiki's[FU] and Donaldson[D0] infinite dimensional symplectic quotient picture. Let $\mathcal{Z}:=\mathcal{J}_{\text {int }}$, the space of integrable almost complex structure on $X$. The tangent space of $\mathcal{J}_{\text {int }}$ at $J$ is given as the following:

$$
T_{J} \mathcal{J}_{\text {int }}:=\{A \in \operatorname{End}(T M) \mid A J+J A=0 \text { and } \omega(A \cdot, \cdot)=\omega(\cdot, A \cdot)\} .
$$

We define the almost complex structure on $\mathcal{J}_{\text {int }}$ as the following:

$$
\begin{gathered}
J_{\mathcal{J}}: T_{J} \mathcal{J}_{\text {int }} \rightarrow T_{J} \mathcal{J}_{\text {int }} \\
J_{\mathcal{J}}(A):=A \cdot J \text { for } A \in T_{J} \mathcal{J}_{\text {int }} ;
\end{gathered}
$$

and the $J_{\mathcal{J}}$-invariant Riemanian metric by

$$
\langle A, B\rangle_{J}:=\int_{X} \operatorname{Tr}(A B) \frac{\omega^{n}}{n !} \text {. for } A, B \in T_{J} \mathcal{J}_{\text {int }}
$$

this makes $\mathcal{J}_{\text {int }}$ a $\infty$-dimensional Kähler manifold.

Now let $G$ be the identity component of the group of exact symplectomorphisms of $(X, \omega)$ then its Lie algebra is given by

$$
\mathfrak{g}=C_{0}^{\infty}(X):=\left\{f \in C^{\infty}(X) \mid \int_{X} f \frac{\omega^{n}}{n !}=0\right\} .
$$

Any function $f$ on $X$ defines a Hamiltonian vector field $\xi_{f}$ on $X$ via

$$
i_{\xi_{f}} \omega=d f
$$

And the symplectic structure $\omega$ defines a Poisson bracket $\{\cdot, \cdot\}$ on $\mathfrak{g}$ via the identity

$$
\{f, g\} \frac{\omega^{n}}{n !}=d f \wedge d g \frac{\omega^{n-1}}{(n-1) !}
$$

and a bi-invariant pairing

$$
\langle f, g\rangle_{\mathfrak{g}^{\mathbb{C}}}:=-\int_{X} f \cdot g \frac{\omega^{n}}{n !} .
$$

Clearly, we have

$$
\langle\{f, h\}, g\rangle_{\mathfrak{g}^{\mathbb{C}}}=\langle f,\{h, g\}\rangle_{\mathfrak{g}^{\mathbb{C}}},
$$

and the natural action of $G$ on $\mathcal{J}_{\text {int }}$ is a holomorphic isometry. Then we have the following theorem due to Donaldson and Fujiki 
Theorem 33. ([D0][FU])The moment map for the action $G$ on $\mathcal{J}_{\text {int }}$ is the scalar curvature. That is

$$
S-s: \mathcal{J}_{\text {int }} \rightarrow \mathfrak{g}=C_{0}^{\infty}(X, \mathbb{R})
$$

given by $S(J)=S(g)$, where $g$ is the Kähler metric on $X$ with $g(\cdot, \cdot)=$ $\omega(\cdot, J \cdot)$ and $s:=\int_{X} S \frac{\omega^{n}}{n !}$.

Now if we want to extend the GIT picture to this infinite dimensional setting then we need to complexify $G$. In order to do so, we complexify $\mathfrak{g}$ as the following:

$$
\mathfrak{g}^{\mathbb{C}}:=\mathfrak{g} \oplus \sqrt{-1} \mathfrak{g}=C_{0}^{\infty}(X, \mathbb{C}) \text { and define* }: f \rightarrow-\bar{f} .
$$

and extend the $\mathfrak{g}$ action on $\mathcal{J}_{\text {int }}$ to a $\mathfrak{g}^{\mathbb{C}}$ action on $\mathcal{J}_{\text {int }}$ via the following

$$
\forall f \in C_{0}^{\infty}(X, \mathbb{R}), \text { we define } \sigma_{J}(i f):=L_{J \xi_{f}} J,
$$

where $L_{J \xi_{f}}$ is the Lie derivative. Then the following lemma enables us to complexify the $G$ action on $\mathcal{J}_{\text {int }}$ on the infinitesimal level (see for instance $[\mathrm{T}])$

\section{Lemma 34.}

$$
\left[L_{\xi} J, L_{J \xi} J\right]=L_{[\xi, J \xi]} J
$$

and

$$
J \circ\left(L_{\xi} J\right):=\left(L_{\xi} J\right) J=\left(L_{J \xi} J\right) .
$$

Remark 35. Note that the complex linear homomorphism

$$
\sigma: \mathfrak{g}^{\mathbb{C}} \longrightarrow T \mathcal{J}_{\text {int }}
$$

is NOT a Lie algbra homomorphism, otherwise, we would be able to conclude from

$$
0=\sigma_{J}\{f, i f\}=\left[L_{\xi} J, L_{J \xi} J\right]=-L_{\left[\xi_{f}, J \xi_{f}\right]} J
$$

that $\xi_{f}$ is a $J$-holomorphic vector field. This implies a reasonable complexification of $G$ does not exist.

Now the Futaki invariant $F_{X}: \mathfrak{a u t}(X, L) \longrightarrow \mathbb{C}$ is defined as following: at any point $J \in \mathcal{J}_{\text {int }}$, we use the Kähler metric $\omega(\cdot, J \cdot)$ to identify aut $(X, L)$ with

$$
\mathfrak{g}_{J}:=\left\{f \in \mathfrak{g}^{\mathbb{C}} \mid \bar{\partial}_{J} \xi_{f}=0, \text { where } i_{\xi_{f}} \omega=d f\right\}
$$


which is a finite dimensional Lie subalgebra of $\mathfrak{g}^{\mathbb{C}}$,then

$$
F_{X}\left(\xi_{f}\right):=\frac{1}{2} \int_{X}(S(J)-s) f \frac{\omega^{n}}{n !}=\frac{1}{2} \int_{X} f \frac{c_{1}(X) \wedge \omega^{n-1}}{(n-1) !}-\frac{s}{2} \int_{X} f \frac{\omega^{n}}{n !}
$$

where

$$
s:=\frac{n \int_{X} c_{1}(X) \wedge \omega^{n-1}}{\int_{X} \omega^{n}}
$$

The lemma above together with Proposition 6 and Corollary 8 give a new proof of the following result of Futaki:

Proposition 36. $F_{X}$ only depends on the Kähler class and $F_{X}$ is a character of $\eta(X)$.

As Donaldson [D0] pointed out the following result originally due to Calabi and Matsushima is a direct consequence of Theorem 10.

Theorem 37. Suppose $J_{0}$ is an extremal point, that is $\omega\left(\cdot, J_{0} \cdot\right)$ is an extremal metric in the sense of Calabi. Let $\mathfrak{g}_{J_{0}}$ denote the Lie algebra of vector field on $X$ which are holomorphic with respect to $J_{0}$. Then we have the following decomposition

$$
\mathfrak{g}_{J_{0}}=\mathfrak{h}_{0}+\bigcup_{\dot{\lambda}>0} \mathfrak{h}_{\lambda}
$$

where

$$
\mathfrak{h}_{\lambda}:=\left\{\xi \in \mathfrak{g}_{J_{0}} \mid\left\{i S\left(J_{0}\right), \xi\right\}=\lambda \cdot \xi\right\}
$$

and $\mathfrak{h}_{0}$ is the reductive part of $\mathfrak{g}_{J_{0}}$. Moreover, we have relations

$$
\left\{\mathfrak{h}_{\lambda_{1}}, \mathfrak{h}_{\lambda_{2}}\right\} \subset \mathfrak{h}_{\lambda_{1}+\lambda_{2}}
$$

and

$$
S\left(J_{0}\right) \in \mathcal{Z}\left(\mathfrak{h}_{0}\right),
$$

where $\mathcal{Z}\left(\mathfrak{h}_{0}\right)$ is the center of $\mathfrak{h}_{0}$. 


\subsection{Finite dimensional picture.}

In this subsection we establish the relation between $f_{X, k}$ and $F_{X}$. Suppose we fix an $S^{1}$-action on $(L, h) \rightarrow X$, that is, where $h$ is a $S^{1}$-invariant Hermitian metrics and $\omega=\frac{\sqrt{-1}}{2 \pi} \operatorname{Ric}(h)$, Then by applying equivariant Riemann-Roch formula the weight $w_{k}$ of the $S^{1}$-action on $\wedge^{\text {top }} H^{0}\left(X, L^{k}\right)$ can be calculated as following

$$
\begin{aligned}
w_{k} & =\left.\frac{d}{d t}\right|_{t=0} \operatorname{ind}\left(e^{t \xi}\right)=\left.\frac{d}{d t}\right|_{t=0} \int_{X} e^{k(\omega+t \mu)} \widetilde{T d} \\
& =\int_{X} k \mu e^{k \omega} T d+\left.\int_{X} e^{k \omega} \frac{d}{d t}\right|_{t=0} \widetilde{T d}
\end{aligned}
$$

where $\mu$ are the moment map associated to $\omega$ with respect to the fixed $S^{1}$-action on $L$ and $\widetilde{T d}$ is the equivariant Todd class. Now if we consider another $S^{1}$-action on $L^{k}$, which is induced from the $k^{t h}$-embedding $X \rightarrow$ $\mathbb{P}^{N_{k}}$, or equivalently we fix an embedding

$$
S^{1} \rightarrow S L\left(N_{k}+1\right) \text {. }
$$

In this case it is clear that weight $\tilde{w}=0$, in particular we have

$$
\begin{aligned}
0 & =\left.\frac{d}{d t}\right|_{t=0} \int_{X} e^{\left(\omega_{F S, k}+t \mu_{F S, k}\right)} \widetilde{T d} \\
& =\int_{X} \mu_{F S, k} e^{\omega_{F S, k}} T d+\left.\int_{X} e^{\omega_{F S, k}} \frac{d}{d t}\right|_{t=0} \widetilde{T d} .
\end{aligned}
$$

Now suppose we have fixed the background Kähler form to be $\omega_{0}$ with corresponding moment map $\mu_{0}$, let $\omega_{1}:=\frac{\omega_{F S}}{k}$, then the moment map $\mu_{1}$ with respect to the original $S^{1}$-action on $L$ is given by $\frac{\mu_{F S, k}}{k}+C$ for some constant $C$. To find $C$, we plug this into the above formula and we deduce

$$
\begin{aligned}
0 & =\left.\frac{d}{d t}\right|_{t=0} \int_{X} e^{k\left(\omega_{1}+t \mu_{1}\right)} \widetilde{T d} \\
& =k \int_{X}\left(\frac{\mu_{F S, k}}{k}+C\right) e^{k \cdot \frac{\omega_{F S, k}}{k}} T d+\left.\int_{X} e^{\omega_{F S, k}} \frac{d}{d t}\right|_{t=0} \widetilde{T d}-C k \int_{X} e^{\omega_{F S, k}} T d \\
& =w_{k}-C k d_{k}
\end{aligned}
$$

where

$$
d_{k}=\operatorname{dim} H^{0}\left(X, L^{k}\right)
$$


This implies

$$
C=\frac{w_{k}}{k d_{k}}
$$

On the other hand, if we normalize $\mu_{0}$ such that $\int_{X} \mu_{0} \frac{\omega_{0}^{n}}{n !}=0$ then we have

$$
\int_{X} \mu_{1} \frac{\omega_{1}^{n}}{n !}=\int_{X} \mu_{0} \frac{\omega_{0}^{n}}{n !}=0
$$

since $\left(\omega_{i}, \mu_{i}\right)$ for $i=1,2$ are with respects to the same $S^{1}$-action on $L$. So

$$
\begin{aligned}
\int_{X} \mu_{F S, k} \frac{\omega_{F S, k}^{n}}{n !} & =k^{n+1} \int_{X}\left(\mu_{1}-C\right) \frac{\omega_{1}^{n}}{n !} \\
& =-\frac{w_{k}}{k d_{k}} k^{n+1} \int_{X} \frac{\omega_{1}^{n}}{n !} \\
& =-k^{n} \int_{X} \mu_{1} \cdot \frac{c_{1}(X) \wedge \omega_{1}^{n-1}}{2(n-1) !}+O\left(k^{n-1}\right)
\end{aligned}
$$

In conclusion, we have proved the following theorem.

\section{Theorem 38.}

$$
\lim _{k \rightarrow \infty} \frac{1}{k^{n}} \int_{X} \mu_{F S, k} \frac{\omega_{F S, k}^{n}}{n !}=-F_{X}
$$

Corollary 39. Suppose for $k$ sufficient large we have

$$
\int_{X} \mu_{F S, k} \frac{\omega_{F S, k}^{n}}{n !}=0
$$

then $F_{X}=0$. In particular, if Chow $\left(X, L^{k}\right)$ is semi-stable for $k>>1$ then $F_{X}=0$

Remark 40. Mabuchi and Nakagawa, J. Ross have obtained similar results in $[M N]$ and $[R]$.

\section{Examples.}

In this section we apply the set up in the previous section to give a simple way of constructing unstable manifold. In particular, by the recent work of Donaldson [D1] these manifolds do not admit constant scalar curvature in the given Kähler class. Our construction is based on the Corollary 9. 


\subsection{Mukai-Tian's example.}

Consider the following exact sequence of vector bundle over the Grassmanian $\mathbb{G}(4,7)$

$$
0 \longrightarrow S^{4} \longrightarrow \mathbb{C}^{7} \longrightarrow Q^{3} \longrightarrow 0
$$

where $S^{4}$ and $Q^{3}$ are universal sub-bundle and universal quotient bundle respectively. Now choose a 3 -dimensional subspace $P \subset \wedge^{2} \mathbb{C}^{7}$, say $P:=$ $\operatorname{span}\left\{u_{1}, u_{2}, u_{3}\right\}$. From the isomorphism

$$
\mathbb{C}^{7} \simeq H^{0}(\mathbb{G}(4,7), Q)
$$

we deduce that each $u_{i}$ can be identified as an element in $H^{0}\left(\mathbb{G}(4,7), \wedge^{2} Q\right)$. So $P:=\left(u_{1}, u_{2}, u_{3}\right)$ can be viewed as a section of the vector bundle

$$
\wedge^{2} Q \oplus \wedge^{2} Q \oplus \wedge^{2} Q \longrightarrow \mathbb{G}(4,7) .
$$

Let $X_{P}:=\left\{P^{-1}(0)\right\} \subset \mathbb{G}(4,7)$. It is not hard to see that for a generic choice of $P \in \mathbb{G}(3,21)=\left\{P^{3} \subset \wedge^{2} \mathbb{C}^{7}\right\}, X_{P}$ is smooth. Our goal is to find the $z_{0}$ in Corollary 9 , to do so we construct a special $P_{0} \in \mathbb{G}(3,21)$. Let

$$
\rho_{n}: S L(2, \mathbb{C}) \rightarrow S L(n, \mathbb{C})
$$

be the irreducible representation of dimension $n$, that is, $\rho_{n}=\rho_{2}^{\otimes(n-1)}$. Then the representation $\rho$ on $\wedge^{2} \mathbb{C}^{7}$ induced by $\rho_{7}$ has decomposition

$$
\rho:=\rho_{3} \oplus \rho_{7} \oplus \rho_{11}
$$

Let $P_{0}$ to be the 3 dimensional invariant subspace corresponding to $\rho_{3}$. If we fix a basis $\left\{e_{i}\right\}_{i=1}^{7}$ for $\mathbb{C}^{7}$ then a straightforward calculation shows that

$$
P_{0}:=\operatorname{span}\left\{u_{1}, u_{2}, u_{3}\right\}
$$

with

$$
\begin{aligned}
& u_{1}:=e_{1} \wedge e_{6}-5 e_{2} \wedge e_{5}+10 e_{3} \wedge e_{4} \\
& u_{2}:=-4 e_{2} \wedge e_{6}+e_{1} \wedge e_{7}+5 e_{3} \wedge e_{5} \\
& u_{3}:=10 e_{4} \wedge e_{5}+e_{2} \wedge e_{7}-5 e_{3} \wedge e_{6} .
\end{aligned}
$$

Notice that $\rho_{7}$ induces a natural action of $S L(2, \mathbb{C})$ on $\mathbb{G}(4,7)$ and

$$
\rho_{7}\left(\left[\begin{array}{cc}
1 & 0 \\
0 & -1
\end{array}\right]\right)=\operatorname{diag}[6,4,2,0,-2,-4,-6] \in S L(7, \mathbb{C}) .
$$


Since all the weights are different the fixed point of $S L(2, \mathbb{C})$ action on $X_{P_{0}}$ must be

$$
\left\{e_{1} \wedge e_{2} \wedge e_{3} \wedge e_{4}, e_{1} \wedge e_{2} \wedge e_{3} \wedge e_{5}, e_{3} \wedge e_{5} \wedge e_{6} \wedge e_{7}, e_{4} \wedge e_{5} \wedge e_{6} \wedge e_{7}\right\}
$$

hence $X_{P_{0}}$ is a homological $\mathbb{P}^{3}$, In particular, it is Fano with Picard number 1. Next we characterize its automorphism.

Claim 5.1. $\mathfrak{a u t}\left(X_{P_{0}}, L\right)=\mathfrak{s l}(2, \mathbb{C})$

Proof. Clearly, we have aut $\left(X_{P_{0}}, L\right) \supseteq \mathfrak{s l}(2, \mathbb{C})$, we need to show aut $\left(X_{P_{0}}, L\right)$ $\subseteq \mathfrak{s l}(2, \mathbb{C})$. Since $X_{P_{0}}$ is Fano, $X_{P_{0}}$ is simply connected which implies $\mathfrak{a u t}\left(X_{P_{0}}, L\right) \subset \mathfrak{s l}(7, \mathbb{C})$ and $P_{0}$ must be the invariant linear subspace for the induced action of $\mathfrak{a u t}\left(X_{P_{0}}, L\right)$ on $\mathbb{C}^{7}$. So $\mathfrak{a u t}\left(X_{P_{0}}, L\right)$ must have a 3 -dimensional irreducible representation which implies that we have homomorphism

$$
\mathfrak{s l}(2, \mathbb{C}) \stackrel{i}{\rightarrow} \mathfrak{a u t}\left(X_{P_{0}}, L\right) \stackrel{j}{\rightarrow} \mathfrak{s l}(2, \mathbb{C})
$$

such that $j \circ i=i d$, this implies $\mathfrak{a u t}\left(X_{P_{0}}, L\right)=\mathfrak{s l}(2, \mathbb{C}) \oplus \mathfrak{k}$. where $\mathfrak{k} \subset \mathfrak{s l}(7, \mathbb{C})$. Since $\mathfrak{k}$ acts trivially on $P_{0}$, it is easy to see that $\mathfrak{k}=0$.

A local calculation at the fixed points of $X_{P_{0}}$ shows that $X_{P_{0}}$ is actually smooth. Since $X_{P_{0}}$ is a homological $\mathbb{P}^{3}, L:=\left.\mathcal{O}_{\mathbb{G}(4,7)}(1)\right|_{X_{P_{0}}} \rightarrow X_{P_{0}}$ is a polarization. For any $k>>1$, the projective embedding of $X_{P_{0}} \hookrightarrow \mathbb{P}^{N_{k}}$ induced by $L^{\otimes k}$ must factor through

$$
X_{P_{0}} \subset \mathbb{G}(4,7) \subset \mathbb{P}^{N_{k}} .
$$

Since $\mathbb{G}(4,7)$ is simply connected, the action of $S L(2, \mathbb{C})$ can be lifted to an action of $S L\left(N_{k}+1, \mathbb{C}\right)$ on $\mathbb{P}^{N_{k}+1}$. Let $\lambda(t):=\exp (\xi t)$ be a one parameter subgroup of $S L(2, \mathbb{C})$ and $P$ be a fixed 3-dimensional linear subspace of $\wedge^{2} \mathbb{C}$ then the degeneration $\lambda(t) \cdot X_{P}$ can be lifted to a degeneration of $X_{P} \subset \mathbb{P}^{N_{k}}$ for any $k$. Now we may apply Corollary 9 to construct examples of unstable manifold, for instance, if we let $\xi:=\operatorname{diag}[6,4,2,0,-2,-4,-6] \in \mathfrak{s l}(2, \mathbb{C})$ and take $P_{a}:=\operatorname{span}\left\{\tilde{u}_{1}, \tilde{u}_{2}, \tilde{u}_{3}\right\}$ as Tian did in $[\mathrm{T}]$ with

$$
\tilde{u}_{i}:=u_{i}+\sum_{l+k \geq 7+i} a_{i l k} e_{l} \wedge e_{k} \text { for } i=1,2,3,
$$

then for generic choice of $\left\{a_{i l k}\right\}, \mathfrak{a u t}\left(X_{P_{a}}\right)=0$. Let $P_{t}:=\exp (\xi t) \cdot P_{a}$ then

$$
\lim _{t \rightarrow \infty} P_{t}=P_{0} .
$$


In particular, this implies

$$
\lim _{t \rightarrow \infty} \lambda(t) \cdot \operatorname{Chow}\left(X_{P_{a}}, L^{k}\right)=\text { Chow }\left(X_{P_{0}}, L^{k}\right) .
$$

Corollary 9 then implies that $X_{P_{a}}$ cannot be balanced, in particular, $X_{P_{a}}$ is not Chow stable for $k>>1$. And Donaldson's theorem [D1] implies that $\left(X_{P_{a}}, L\right)$ do not admit constant scalar curvature metric.

Notice that the above construction can be applied to more general situation, a very simple way of constructing unstable manifolds is taking a small projective deformation of a projective manifold $X_{0}$ with aut $(X)$ being simple, e.g. a deformation of $\mathbb{P}^{r} \times \Sigma$, where $\Sigma$ is a Kähler manifold with $c_{1}(\Sigma)<0$. (Note that Burns and De Bartolomeis' example [BD] is a special case of this sort) This construction is closely related to the work of LeBrun and Simanca [LS].

$$
\text { 5.2. } \mathbb{P}^{2} \# \overline{\mathbb{P}^{2}} \text {. }
$$

Let $X$ be the Steiner surface $X \hookrightarrow \mathbb{P}^{4}$ defined by image of the following birational map

$$
\begin{array}{ccc}
\mathbb{P}^{2} & -\rightarrow & \mathbb{P}^{4} \\
{[x, y, z]} & \longrightarrow & {\left[x z, y z, x^{2}, x y, y^{2}\right]}
\end{array}
$$

$X$ is $\mathbb{P}^{2}$ blowing up the point $[0: 0: 1]$. Then

$$
\mathcal{O}_{X}(1):=\left.\mathcal{O}_{\mathbb{P}^{4}}(1)\right|_{X}=2 H-E,
$$

where $H$ is the hyperplane class and $E$ is the exceptional divisor. And

$$
\begin{gathered}
H^{0}\left(X, \mathcal{O}_{X}(m)\right)=\left\{x^{i} y^{j} z^{k} \mid i+j+k=2 m \text { and } k \leq m\right\}, \\
d_{m}:=\operatorname{dim} H^{0}\left(X, \mathcal{O}_{X}(m)\right)=\frac{1}{2}\left(3 m^{2}+5 m+2\right) .
\end{gathered}
$$

Let $\xi=\operatorname{diag}\left[\lambda_{1}, \lambda_{2}, \lambda_{3}\right] \in \mathfrak{s l}(3)$, then $e^{\xi t}-$ action on $X$ gives rise to an action on the $m^{\text {th }}$-embedding $X \stackrel{\mathcal{O}_{X}(m)}{\longrightarrow} \mathbb{P}^{d_{m}-1}$. If we normalize the action to be special linear then the weight of $x^{i} y^{j} z^{k} \in H^{0}\left(X, \mathcal{O}_{X}(m)\right)$ is $\lambda_{1} i+\lambda_{2} j+\lambda_{3} k-\bar{\lambda}$ with

$$
\begin{aligned}
\bar{\lambda} & =\frac{1}{d_{m}} \sum_{\substack{i+j+k=2 m \\
k \leq m}} \lambda_{1} i+\lambda_{2} j+\lambda_{3} k \\
& =\frac{-\lambda_{3} m(m+1)}{(3 m+2)} .
\end{aligned}
$$


Let $L \rightarrow X$ to be the pull back of $\mathcal{O}_{\mathbb{P}^{d_{m}-1}}(1)$, then the weight $w_{m, k}$ on $\wedge^{t o p} H^{0}\left(X, L^{k}\right)$ is given by

$$
\begin{aligned}
w_{m, k} & =\sum_{\substack{i+j+l=2 m k \\
l \leq k m}}\left(\lambda_{1} i+\lambda_{2} j+\lambda_{3} l-k \bar{\lambda}\right) \\
& =\lambda_{3} m^{2}\left(\frac{1}{6}+O\left(m^{-1}\right)\right) k^{3}+O\left(k^{2}\right)
\end{aligned}
$$

and

$$
\begin{aligned}
\frac{w_{k}}{k d_{k}} & =\frac{-\lambda_{3}(k+1)^{2}}{2 d_{k}}-\bar{\lambda} \\
& =\frac{-2 \lambda_{3}}{3}-\frac{\lambda_{3}}{9} \frac{1}{k}-\bar{\lambda}+O\left(\frac{1}{k^{2}}\right),
\end{aligned}
$$

where $w_{k}$ is the weight of $\xi$ on $\wedge^{t o p} H^{0}\left(X, \mathcal{O}_{X}(k)\right)$ and

$$
d_{k}=\frac{1}{2}\left(3 k^{2}+5 k+2\right)=\frac{1}{2}(3 k+2)(k+1) .
$$

Then we find

$$
f_{X, m}=\text { leading coefficient of } w_{m, k}=\frac{\lambda_{3} m^{2}}{6}\left(1-\frac{2}{3 m}+O\left(m^{-2}\right)\right)
$$

and by Theorem 38, we see that the Futaki invariant of $\xi$

$$
F_{X}(\xi)=-\lim _{m \rightarrow \infty} \frac{f_{X, m}}{m^{2}}=-\frac{\lambda_{3}}{6}=-\frac{\lambda_{3}}{9} \int_{X} \frac{\omega^{2}}{2 !},
$$

which agrees with Donaldson's definition of Futaki invariant in [D2].

\section{References.}

[BD] D. Burns and P. De Bartolomeis Stability of vector bundles and extremal metrics. Invent. Math. 92 (1988), no. 2, 403-407.

[D0] S.K. Donaldson, Remarks on Gauge theory, Complex Geometry and 4-manifold Topology, Fields Medallists' lectures, 384-403, World Sci. Ser. 20th Century Math,.5, World Sci. Publishing.

[D1] S.K. Donaldson, Scalar curvature and projective embeddings, I, J. Diff. Geom. 59(2001), no., 479-522. 
[D2] S.K. Donaldson, Scalar curvature and stability of toric varieties. J. Diff. Geom. 62 (2002), no. 2, 289-349.

[DK] S.K. Donaldson and P.B. Kronheimer, The Geometry of FourManifolds, Clarendon,Oxford 1990.

[F] A. Futaki, Kähler-Einstein metrics and integral invariants, Lecture Notes in Mathematics, 1314. Springer-Verlag, Berlin, 1988.

[FM1] A. Futaki and T. Mabuchi, Bilinear forms and extremal Kähler vector fields associated with Kähler classes, Math. Ann. 301 (1995), no. 2, 199-210.

[FM2] A. Futaki and T. Mabuchi, Moment maps and symmetric multilinear forms associated with symplectic classes, Asian J. Math. 6 (2002), no. $2,349-371$.

[FU] A. Fujiki, The moduli spaces and Kähler metrics of polarized algebraic varieties. (Japanese) Sugaku 42 (1990), no.3, 231-243.

[G] D. Gieseker, Lectures on moduli of curves, TATA Note series. 68(1982), New York. Springer-Verlag.

[HA] R. Hartshorne, Algebraic Geometry, Springer-Verlag 1977.

[HL] D. Huybrechts and M. Lehn, The Geometry of Moduli Spaces of Sheaves, Vieweg 1997.

[K] F. Kirwan, Cohomology of Quotients in Symplectic and Algebraic Geometry, Princeton 1984.

[Luo] H. Luo, Geometric criterion for Gieseker-Mumford stability of polarized manifolds, J. Diff. Geom. 49(1998), no. 3, 577-599.

[LS] C. LeBrun and S. R. Simanca Extremal Kähler metrics and complex deformation theory, G.A.F.A. 4(1994), no. 3, 298-336.

[M] T. Mabuchi, An algebraic character associated with the Poisson brackets. Recent topics in differential and analytic geometry, 339358, Adv. Stud. Pure Math., 18-I, Academic Press, Boston, MA, 1990 .

[MFK] D. Mumford, J. Fogarty and F. Kirwan, Geometric Invariant Theory, Springer Verlag,Berlin (1994). 
[MN] T. Mabuchi and Y. Nakagawa, The Bando-Calabi-Futaki character as an obstruction to semistability, Math. Ann. 324 (2002), no. 1, 187-193.

[Mum] D. Mumford, Stability of Projective Varieties, L'Enseignement Mathématique 1977.

[P] S. Paul Geometric analysis of Chow Mumford stability Princeton Ph.D. Thesis. (2000).

[R] J. Ross Instability of polarised algebraic varieties Imperial College Ph.D. Thesis. (2003)

[T] G. Tian Canonical Metrics in Kähler Geometry, Birkhäuser,(2000)

[W] X. Wang Balance point and stability of vector bundles over a projective manifold, Math. Res. Lett. 9 (2002), no. 2-3, 393-411.

[Z] S. Zhang, Heights and reductions of semi-stable varieties. Compositio Math. 104 (1996), no. 1, 77-105.

UCLA MATH.

P.O. Box 951555

Los ANGELEs, CA 90095-1555

xiaowei@math. ucla.edu

Received February 23, 2004. 
